\title{
The Survey of the Patient Received the Epiduroscopic Laser Neural Decompression
}

\author{
Department of Anesthesiology and Pain Medicine, Konyang University Hospital, Daejeon, Korea
}

Dae Hyun Jo, MD, and Hun Ju Yang, MD

\section{Background:}

Neuroplasty using a Racz catheter or epiduroscope and percutaneous endoscopic laser discectomy are performed as treatment for chronic refractory low back and/or lower extremity pain, but they are limited in that they cannot completely remove the causing pathology. Lately, epiduroscopic laser neural decompression (ELND) has been receiving attention as an alternative treatment, but there are insufficient reports of results. Hence we aimed to investigate and report the data in our hospital.

\section{Methods:}

Seventy-seven patients were selected who had received ELND via the anterior and posterior epidural approach through the pain clinic in our hospital from March 2011 to July 2012. Their medical records including age, diagnosis, epiduroscopic findings and degree of symptom relief were investigated. The degree of symptom relief following the procedure was categorized into 5 stages of very good (5), good (4), no change (3), bad (2), and very bad (1) at 2 weeks and 1 month after the procedure.

\section{Results:}

The subjects were 30 males and 47 females. Mean age was 54.6 for males and 59.6 for females, so the overall mean age was 58.1 years old, with the youngest being 23 and the oldest 88 years old. In epiduroscopic images of all patients, more than one situation of herniated disc, fibrous tissue and adhesion, or inflammation was observed. Sixty-seven patients (87.0\%) showed symptom relief 2 weeks after the procedure and 63 patients (81.8\%) showed relief after 1 month.

\section{Conclusions:}

ELND is considered to be an effective treatment alternative for chronic refractory low back and/or lower extremity pain, including lumbar disc herniation, lumbar spinal stenosis, and failed back surgery syndrome which cannot be alleviated with existing non-invasive conservative treatment. (Korean J Pain 2013; 26: 27-31)

\section{Key Words:}

anterior epidural approach, epiduroscopic laser neural decompression.

Received August 22, 2012. Revised October 27, 2012. Accepted October 29, 2012.

Correspondence to: Hun Ju Yang, MD

Department of Anesthesiology and Pain Medicine, Konyang University Hospital, 158 Gwanjeodong-ro, Seo-gu, Daejeon 371-718, Korea Tel: +82-42-600-9294, Fax: +82-42-600-9090, E-mail: yusyuni@daum.net

() This is an open-access article distributed under the terms of the Creative Commons Attribution Non-Commercial License (http:// creativecommons.org/licenses/by-nc/3.0/), which permits unrestricted non-commercial use, distribution, and reproduction in any medium, provided the original work is properly cited.

Copyright (c) The Korean Pain Society, 2013 


\section{INTRODUCTION}

Chronic refractory low back and/or lower extremity pain that is not alleviated by non-invasive conservative treatment including fluoroscopically-directed epidural steroid injection is bewildering for the patient and the doctor [1-4]. Neuroplasty using a Racz catheter or epiduroscopic treatment can be helpful to patients who do not respond to conservative treatment as well as failed back surgery syndrome (FBSS) patients, but it is limited in that the related pathology cannot be removed completely. However, recently laser ablation using epiduroscope became possible due to the development of a two working channel system of video guided catheter and subsequent application of an optical laser fiber, and hence a new chapter was opened in the treatment of patients with chronic refractory low back and/or lower extremity pain $[5,6]$.

Epiduroscopic laser neural decompression (ELND) maintains the advantages of the existing epiduroscopic adhesiolysis, such as non-invasiveness, short operating time, reduction of risks related to general anesthesia, possibility of communication with the patient during the procedure (leading to reduced risk of accidental nerve damage), and short recovery time. In addition, it has the advantage that the causing pathology can be treated directly, by reducing the volume of the herniated disc in the affected area and direct removal of severely adhered areas with the laser. In other words, not only can the cause of the pain be directly observed through the epiduroscope, but treatment of the lesion can also be performed at the time of diagnosis. This can reduce the burden of repeated surgery for FBSS patients, and even patients with lesions in various lumbar segments can be treated with only a single insertion of the epiduroscope.

However, ELND is in its initial stage, so there are almost no clinical reports of results. Hence the authors conducted a retrospective review of medical records of $\mathrm{pa}^{-}$ tients who had received ELND in our hospital, to report the results of the procedure and determine its clinical significance.

\section{MATERIALS AND METHODS}

A retrospective follow-up investigation was conducted on the medical records of 77 patients who received ELND at the pain clinic of our hospital from March 2011 to July
2012. The procedure was performed on patients complaining of refractory low back and/or lower extremity pain which did not improve despite other non-invasive conservative treatment including fluoroscopically-directed epidural steroid injection, or in cases of which $50 \%$ or more of the pain returned within 1 week. Cases of low back and/or lower extremity pain which could not be clearly explained with MRI were also included for the procedure.

While reviewing the medical records of all patients in the study, the lumbar MRI images were also examined, and epiduroscopic images at the time of the procedure were reviewed to observe the lesions inside the epidural space. The age, sex, disease diagnosed to be causing the pain, duration of the disease, and degree of pain alleviation after the procedure were investigated for all patients. The duration of the disease was expressed as years and months. Degree of pain alleviation was categorized into 5 stages: very good (5), good (4), no change (3), bad (2), and very bad (1) on the basis of the patients' comments recorded in the medical records 2 weeks and 1 month after the procedure.

\section{RESULTS}

The patient subjects were 30 males and 47 females, and the mean age was 54.6 years for males and 59.6 years for females. The mean age of all subjects was 58.1 years, with the youngest patient being 23 and oldest 88 years old.

When the entire patient group was categorized according to diagnosis by MRI finding, there were 4 patients with FBSS, 44 patients with lumbar disc herniation, $11 \mathrm{pa}-$ tients with lumbar spinal stenosis, 16 patients with lumbar disc herniation and lumbar spinal stenosis, and 2 patients with lumbar facet joint syndrome. Regarding the duration of the symptom prior to the procedure, the time was less than 3 months for 14 patients, 3 months or more but less than 6 months for 8 patients, 6 months or more but less than 1 year for 8 patients, 1 year or more but less than 5 years for 23 patients, and 5 years or more for 24 patients.

In epiduroscopic images of all patients, lesions such as fibrous bands, adhesions, scar tissue, and inflammation were observed, and additional protruded or extruded disc lesions could be observed in patients with lumbar disc herniation or lumbar disc herniation accompanying lumbar spinal stenosis.

When the symptom relief scores according to fol- 


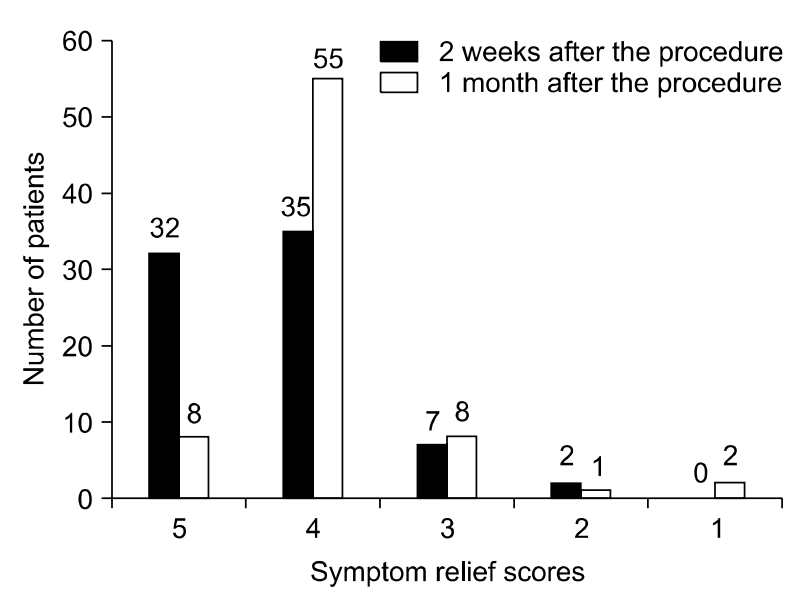

Fig. 1. The symptom relief scores according to follow-up period after the procedure.

low-up period after the procedure were examined, $81.8 \%$ of all patients were found to have improved 1 month after the procedure (Fig. 1).

One patient did not follow up 2 weeks after the procedure, and 3 patients did not follow up 1 month after the procedure.

\section{DISCUSSION}

In patients complaining of chronic refractory low back and/or lower extremity pain despite non-invasive conservative treatment, there should be suspicion of lumbar disc herniation, lumbar spinal stenosis, failed back surgery syndrome, sacroiliac joint syndrome, and even epidural fibrosis as the cause. Epidural fibrosis develops from the dense fibrous tissue infiltrating a hematoma which develops after a surgical procedure, and it can also develop secondarily without a surgical operation from annular tear, hematoma, infection, or even intrathecal contrast media [1]. Such epidural fibrosis, as well as adhesions and scar tissue, change the normal fat cells in the epidural space into fibrous tissue, and attach the nerve roots to surrounding structures. Nerve fibers are surrounded by scar tissue, which leads to increased tension as well as damage of axoplasmic transport, and causes ischemic pain from limited blood supply. The lumbar nerve roots and dorsal root ganglion are sensitive to mechanical changes, so compression of nerve tissues causes symptoms such as pain, muscle weakness, and numbness [7]. Epidural adhesiolysis is widely performed to treat the pain by blocking nerve activity in the scar tissue area within the epidural space and relieving adhesions by reducing inflammation and edema. However, it is limited in that it cannot solve the pathology of dense fibrous tissue or protruded disc tissue. On the other hand, ELND removes adhesions surrounding nerve roots while directly observing the epidural space with an epiduroscope. Medication is directly injected around the lesion to reduce pain, and a laser fiber can be inserted through the working channel to directly remove the lesion area such as fibrous bands, protruded disc, etc. with the laser.

The Holmium: YAG (Ho: YAG) laser used by the authors has a wavelength of 2,100 nm and produces almost no bleeding either during or after the procedure as it absorbs better into the water within the tissue than into blood vessels. The depth of infiltration into the tissue is limited to around $0.3-0.5 \mathrm{~mm}$ so accurate cutting is possible and the risk of damaging tissue surrounding the target area is reduced. The laser also minimizes complications as it does not affect surrounding blood vessels or nerves. Hence it is widely used clinically. Also, in contrast to the existing CO2 laser, the pulsed energy conveyed by the Holmium laser minimizes thermal damage and allows fiberoptic delivery, so it is ideal to use in endoscopic procedures [8].

Due to such characteristics of the laser, the risk of re-adhesion due to scar tissue and fibrosis caused by post-procedural bleeding was substantially reduced [9]. The minimization of surrounding tissue damage also led to minimization of the possibility of recurrence of disc herniation or instability after the procedure, which had been argued by Marchetti et al. [10] as the only indication for repeating surgery. However, motor nerve paralysis caused by thermal damage of nerve roots, local sensory impairment, fiber breakage, and discitis have been reported as complications related to laser treatment, so particular caution is necessary during the laser procedure [11].

Epiduroscopy is a minimally invasive procedure, but it can cause pain upon insertion of the dilator and introducer sheath, and paresthesia and headache during the procedure [12]. Complications that can occur after the procedure are temporary aggravation of pain, subcutaneous infection, epidural abscess, arachnoiditis, meningitis, paresthesia and paralysis, paraplegia, nerve root damage, postdural puncture headache, blood vessel damage and injection of medication into blood vessel, cerebral embolism, and pulmonary embolism [12]. In addition, normal saline is 

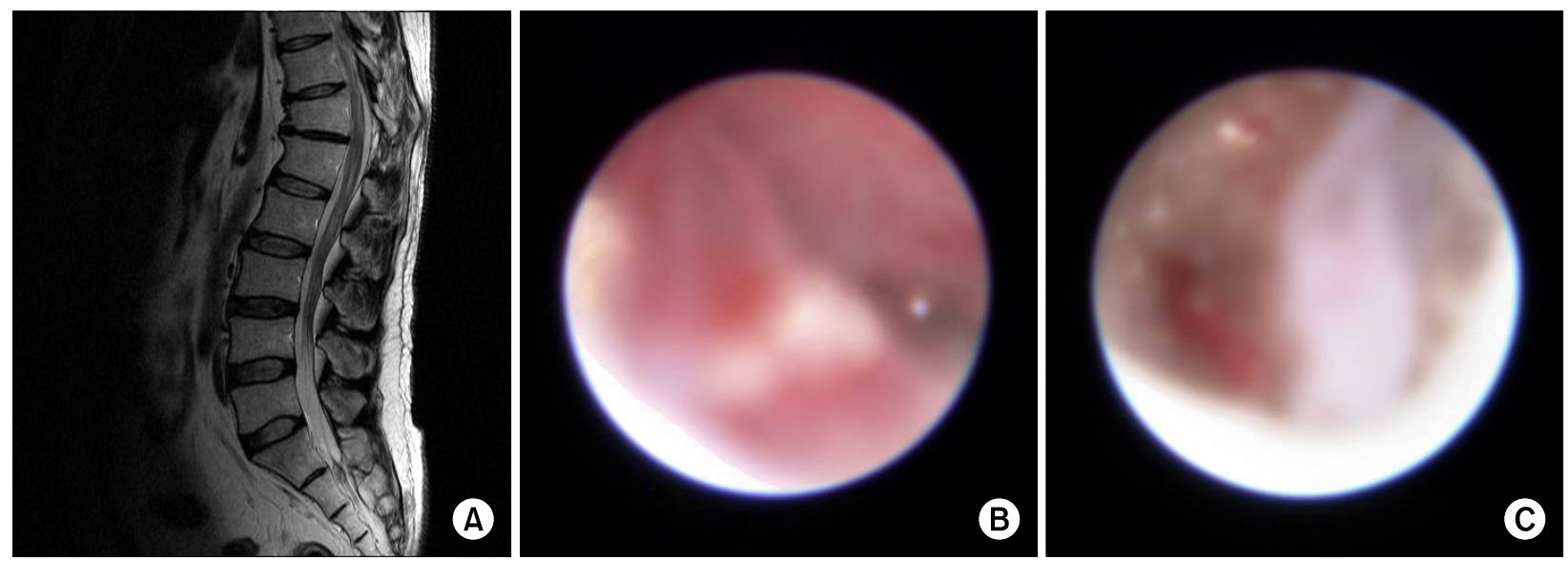

Fig. 2. A 44-year-old man diagnosed of facet joint syndrome at L4-5, L5-S1. (A) T2-weighted MR sagittal image which cannot show epidural fibrosis. (B, C) Epiduroscopic finding of inflammation (B) and thick fibrotic band lesion (C).

injected for epidural irrigation, expansion, and to ensure the field of vision, which can heighten epidural pressure and cause pain, and even lead to excessive intracranial hemorrhage and retinal hemorrhage [2]. Therefore, if the patient complains of pain during the procedure, the injection must be stopped immediately and epidural pressure must be lowered. The authors ensured that the total amount of normal saline injected did not exceed $200 \mathrm{ml}$ in any of the patients in the study. During this investigation, there was one patient whose sutured area opened after removal of the stitches from the operated area 1 week after the procedure. This suggests that caution is necessary in stitching the operated area after the procedure, as well as in subsequent management of the sutured area.

This investigative study included patients complaining of low back and/or lower extremity pain which could not be clearly explained by MRI. The two patients with lumbar facet joint syndrome had not shown satisfactory alleviation of symptoms after medial branch block or facet joint injection. However, after the performance of ELND, one of the patients experienced symptom relief. Epidural fibrosis could not be discovered with CT or MRI, so the epiduroscope was used in such patients to evaluate the morbid changes firsthand, and simultaneously remove fibrous tissue and inject medication for treatment (Fig. 2). In other words, the epiduroscope is useful as a tool to diagnose the cause of low back and/or lower extremity pain as well as a tool for treatment. Of course there is controversy about whether to apply epiduroscopy to all patients with chronic refractory low back and/or lower extremity pain which does not correspond with CT or MRI findings. Also, there has been no attempt to evaluate the usefulness of the epiduroscope for diagnostic purposes due to problems of high cost. When the problems of high cost are solved and the epiduroscope can be freely utilized for diagnostic purposes, ELND could be applied more widely to patients with chronic refractory low back and/or lower extremity pain. There are no established indications yet, so additional prospective study data should be accumulated.

According to the data in our investigation, inserting the epiduroscope into the front of the epidural space to confirm the lesion in the anterior epidural space and treat it with laser resulted in symptom relief in $87 \%$ of the patients 2 weeks after the procedure and in $81.8 \%$ of the patients 1 month after the procedure. This investigation is only the evaluation results of a period of up to 1 month after the procedure, so there are questions regarding the results of long-term follow-up observation. As this data derives from attempts at a new procedure, it is considered that supplementation is required through further continuous observation.

Considering the results of this investigation, the authors anticipate that ELND can be an effective treatment alternative for patients with chronic refractory low back and/or lower extremity pain that cannot be solved by other non-invasive conservative treatments, including the existing fluoroscopically-directed epidural steroid injection. 


\section{REFERENCES}

1. Manchikanti L, Boswell MV, Rivera JJ, Pampati VS, Damron $\mathrm{KS}$, McManus $C D$, et al. A randomized, controlled trial of spinal endoscopic adhesiolysis in chronic refractory low back and lower extremity pain [ISRCTN 16558617]. BMC Anesthesiol 2005; 5: 10.

2. Hayek SM, Helm S, Benyamin RM, Singh V, Bryce DA, Smith HS. Effectiveness of spinal endoscopic adhesiolysis in post lumbar surgery syndrome: a systematic review. Pain Physician 2009; 12: 419-35.

3. Manchikanti L, Saini B, Singh V. Spinal endoscopy and lysis of epidural adhesions in the management of chronic low back pain. Pain Physician 2001; 4: 240-65.

4. Manchikanti L, Rivera JJ, Pampati V, Damron KS, Beyer CD, Brandon DE, et al. Spinal endoscopic adhesiolysis in the management of chronic low back pain: a preliminary report of a randomized, double-blind trial. Pain Physician 2003; 6: 259-67.

5. Richter EO, Abramova MV, Cantu F, De Andres J, Lierz P, Manchiaro PL, et al. Anterior epiduroscopic neural decompression: eight-center experience in 154 patients. Eur $\mathrm{J}$
Pain Suppl 2011; 5: 401-7.

6. Schenk B, Brouwer PA, van Buchem MA. Experimental basis of percutaneous laser disc decompression (PLDD): a review of literature. Lasers Med Sci 2006; 21: 245-9.

7. Lee SI, Kim KT, Hwang JK. Endoscopic and non-endoscopic epidural adhesiolysis in FBSS patient. Korean $J$ Anesthesiol 2004; 46: 329-35.

8. Ruetten S, Meyer O, Godolias G. Application of holmium:YAG laser in epiduroscopy: extended practicabilities in the treatment of chronic back pain syndrome. J Clin Laser Med Surg 2002; 20: 203-6.

9. Epstein JM. Adler R. Laser-assisted percutaneous endoscopic neurolysis. Pain Physician 2000; 3: 43-5.

10. Marchetti PG, Binazzi R, Vaccari V, De Zerbi M, Landi S. Failed back syndromes: opinions and personal experiences. Chir Organi Mov 1994; 79: 127-30.

11. Ahn JS. Laser decompression. J Korean Soc Spine Surg 2000; 7: 318-21.

12. Lee $W Y$, Jeong $Y S$. The effects of epiduroscopy in spinal stenosis: a case report. J Korean Pain Soc 2002; 15 : 154-8. 